\title{
Trigeminal neuralgia without neurovascular compression presents earlier than trigeminal neuralgia with neurovascular compression
}

\author{
Andrew L. Ko, MD, ${ }^{1}$ Albert Lee, MD, ${ }^{2}$ Ahmed M. Raslan, MD, ${ }^{1}$ Alp Ozpinar, BA, ${ }^{1}$ \\ Shirley McCartney, PhD, ${ }^{1}$ and Kim J. Burchiel, MD1 \\ 1Department of Neurological Surgery, Oregon Health \& Science University, Portland, Oregon; and 2Department of Neurological \\ Surgery, University of Indiana, Indianapolis, Indiana
}

\begin{abstract}
OBJECT Trigeminal neuralgia (TN) occurs and recurs in the absence of neurovascular compression (NVC). To characterize what may be distinct patient populations, the authors examined age at onset in patients with $\mathrm{TN}$ with and without NVC.

METHODS A retrospective review of patients undergoing posterior fossa surgery for Type I TN at Oregon Health \& Science University from 2009 to 2013 was undertaken. Charts were reviewed, and imaging and operative data were collected for patients with and without NVC. Mean, median, and the empirical cumulative distribution of onset age were determined. Statistical analysis was performed using Student t-test, Wilcoxon and Kolmogorov-Smirnoff tests, and Kaplan-Meier analysis. Multivariate analysis was performed using a Cox proportional hazards model.

RESULTS The charts of 219 patients with TN were reviewed. There were 156 patients who underwent posterior fossa exploration and microvascular decompression or internal neurolysis: 129 patients with NVC and 27 without NVC. Mean age at symptoms onset for patients with and without NVC was 51.1 and 42.6 years, respectively. This difference (8.4 years) was significant (t-test: $p=0.007$ ), with sufficient power to detect an effect size of 8.2 years. Median age between groups with and without NVC was 53.25 and 41.2 years, respectively $(p=0.003)$. Histogram analysis revealed a bimodal age at onset in patients without NVC, and cumulative distribution of age at onset revealed an earlier presentation of symptoms $(p=0.003)$ in patients without NVC. Chi-square analysis revealed a trend toward female predominance in patients without NVC, which was not significant $(p=0.08)$. Multivariate analysis revealed that age at onset was related to NVC but not sex, symptom side or distribution, or patient response to medical treatment.

CONCLUSIONS NVC is neither sufficient nor necessary for the development of TN. Patients with TN without NVC may represent a distinct population of younger, predominantly female patients. Further research into the pathophysiology underlying this debilitating disease is needed.

http://thejns.org/doi/abs/10.3171/2014.11.JNS141741
\end{abstract}

KEY WORDS trigeminal neuralgia; neurovascular compression; age at symptom onset; microvascular decompression; peripheral nerve; pain

I N 1934, Dandy proposed neurovascular compression (NVC) as an etiology for trigeminal neuralgia (TN). In his series, he reported that the superior cerebellar artery caused NVC in $30.7 \%$ of patients. ${ }^{6}$ He noted veins, tumors, and vascular and cranial malformations as other "gross findings" causing compression of the trigeminal nerve; nevertheless, in $40 \%$ of cases, he observed no evidence of a gross lesion. ${ }^{6} \mathrm{He}$ did document, however, that his posterior fossa approach did not allow for visualization of the entire root entry zone (REZ).

After years of observation, and an autopsy series that showed no NVC in cadavers without $\mathrm{TN},{ }^{13}$ Jannetta advanced the hypothesis that TN was a "nerve entrapment syndrome," resulting from arteries both lengthened and rendered tortuous by arteriosclerotic cerebrovascular disease. ${ }^{13}$ This putative mechanism accounted for recurrence

ABBREVIATIONS AED = antiepileptic drug; IN = internal neurolysis; $M S$ = multiple sclerosis; $M$ VD = microvascular decompression; NVC = neurovascular compression; $\mathrm{OHSU}=$ Oregon Health \& Science University; REZ = root entry zone; TN = trigeminal neuralgia; TN-I = Type I TN; TN-II = Type II TN.

SUBMITTED July 29, 2014. ACCEPTED November 12, 2014.

INCLUDE WHEN CITING Published online June 5, 2015; DOI: 10.3171/2014.11.JNS141741.

DISCLOSURE The authors report no conflict of interest concerning the materials or methods used in this study or the findings specified in this paper. 
of symptoms by positing that the continued lengthening of the offending artery could, over time, recompress the trigeminal nerve at the pons. ${ }^{13}$

The unequivocal success of microvascular decompression (MVD) in treating TN has supported the role of NVC in TN pathophysiology. Furthermore, the presence and severity of NVC have been shown to impact the long-term results of MVD. ${ }^{1,4,32}$ However, it is increasingly clear that $\mathrm{TN}$ occurs and recurs in the absence of $\mathrm{NVC},{ }^{19}$ and that NVC of the trigeminal nerve exists in a sizeable population without TN..$^{25}$ Thus, NVC is neither a necessary nor sufficient condition for the development of TN. Nevertheless, NVC does play a role in disease pathogenesis.

There is sufficient evidence that TN is not a homogeneous disorder. For example, it is well accepted that Type I TN (TN-I) and Type II TN (TN-II) have distinct natural histories and responses to MVD treatment. ${ }^{24,26}$ The presence or absence of NVC may likewise reflect distinct patient populations with $\mathrm{TN}$.

The aim of this study was to examine a large population of patients with TN with imaging and surgical confirmation of NVC treated with posterior fossa exploration and MVD or internal neurolysis (IN). The objective of the present study was to characterize potential differences in patients with TN with and without NVC.

\section{Methods \\ Study Design and Diagnostic Criteria}

Retrospective review of patients 18 years of age or older with TN-I or TN-II treated at Oregon Health \& Science University (OHSU) from July 2006 to February 2013 was undertaken. The study was approved by the OHSU Institutional Review Board.

The diagnostic and treatment algorithm for facial pain is standardized at OHSU. Diagnosis was made using clinical interview and examination. ${ }^{19,23,24}$ Using the Burchiel ${ }^{3}$ classification of facial pain, all patients had TN-I, characterized by idiopathic, episodic, lancinating pain lasting several seconds, with pain-free intervals between attacks. Importantly, these patients did not have what is described as TN-II, which is predominantly ( $>50 \%$ of the time) aching, throbbing, or burning pain, with a minor component of lancinating pain. This is a clinical diagnosis, and hence all diagnoses were made during initial evaluation, prior to advanced imaging and prior to findings during surgery. Of note, using the International Classification of Headache Disorders (3rd edition), all patients described here would have been classified as classical TN (13.1.1.1 TN developing without apparent cause other than NVC, purely paroxysmal) or as classical TN with concomitant persistent pain (13.1.1.2); this classification system does not emphasize the predominance of persistent pain that characterizes TN-II by the Burchiel classification. ${ }^{12}$ All patients with TN-I were screened for signs, symptoms, or family history of multiple sclerosis (MS). No patient with such indication or history was considered to have a diagnosis of TN I. None of the patients showed evidence of demyelinating disease on subsequent imaging.

Barring the presence of severe medical comorbidities or very advanced age, patients were offered MVD as first- line treatment. All patients who were considered candidates for MVD underwent evaluation with high-resolution MRI/MR angiography, and the images were 3-dimensionally reconstructed and evaluated for NVC as previously described. ${ }^{25}$ Patients with NVC underwent craniotomy and MVD; patients without NVC underwent craniotomy and IN. That is, imaging findings did not change the treatment algorithm with respect to whether patients underwent posterior fossa exploration.

Chart review was performed to assess demographic and surgical data for all patients. In particular, age at symptom onset and age at presentation to clinic were confirmed for all patients. Review of imaging data and intraoperative videos was performed to confirm presence or absence of NVC.

The final determination of whether a patient had NVC or not was made by the senior author and the surgical fellow, based on operative findings and confirmed by subsequent intraoperative video review.

\section{Statistical Analysis}

Statistical analysis was performed using commercially available software (MATLAB). Analysis of means was performed using the Student t-test, and power calculations were performed. Wilcoxon's rank-sum test was used to compare median values. Chi-square analysis was applied for categorical comparisons. Cumulative distributions were compared using the 2-sample Kolmogorov-Smirnov test. Kaplan-Meier statistics were calculated for patients having onset of TN symptoms with NVC versus those without NVC. Multivariate analysis was also performed using a Cox proportional hazards model for demographic and clinical factors possibly contributing to earlier age at onset of TN symptoms. Data are presented \pm standard deviation.

\section{Results}

\section{Demographics and Patient Characteristics}

A review of the imaging data obtained during the 5-year study period for patients with TN-I $(n=219)$ revealed that 61 patients were without NVC and 158 with NVC; 156 patients with TN-I underwent posterior fossa exploration and MVD or IN. Of the 156 surgical patients, there were 129 with (83\%) and 27 without (17\%) NVC (taking into account prior procedures, some of which were performed at outside institutions). These patients were assumed to have compression at their initial surgery if evidence of decompression was seen during reoperation. A total of 15 patients underwent previous exploration at an outside hospital. Of these, 9 had imaging evidence of NVC, 1 had evidence of NVC at exploration but not on imaging, and 5 had no evidence of NVC. Those with NVC had either recurrence or inadequate decompression at their first surgery. Those without NVC showed no evidence of previous decompression at reoperation (i.e., no Teflon).

Demographic data for patients in this series are presented in Table 1 . There was no significant difference in the laterality, sex, or distribution of pain symptoms between patients with or without NVC. The proportion of patients who responded to treatment with antiepileptic 
TABLE 1. Demographics and characteristics of patients with TN

\begin{tabular}{|c|c|c|c|}
\hline Characteristic & w/ NVC & w/o NVC & Total \\
\hline \multicolumn{4}{|l|}{ Sex (no.) } \\
\hline M & 57 & 7 & 64 \\
\hline $\mathrm{F}$ & 72 & 20 & 92 \\
\hline Total & 129 & 27 & 156 \\
\hline$p$ value ( $\chi^{2}$ value) & & & $0.08(3.08)$ \\
\hline \multicolumn{4}{|l|}{ Symptom side (no., expected $\chi^{2}$ value) } \\
\hline $\mathrm{Rt}$ & $66,64.5$ & $12,13.5$ & 78 \\
\hline Lt & $60,62.0$ & $15,12.9$ & 75 \\
\hline Bilat & $3,2.4$ & $0,0.5$ & 3 \\
\hline Total & 129 & 27 & 156 \\
\hline$p$ value ( $\chi^{2}$ value $)$ & & & $0.54(1.21)$ \\
\hline \multicolumn{4}{|l|}{ Symptom duration (mos) } \\
\hline Mean & 72.2 & 43.3 & $p=0.07$ \\
\hline Median & 45 & 36 & $p=0.32$ \\
\hline Total & Mean $67.2 \pm 77.7$ & Median 45 & Range $0.1-450$ \\
\hline Patients with previous procedures (no., \%) & 18,14 & $8,29.6$ & $26,16.7$ \\
\hline$p$ value ( $\chi^{2}$ value $)$ & & & $0.05(3.95)$ \\
\hline \multicolumn{4}{|l|}{ Previous procedures (no.) } \\
\hline Posterior fossa & 15 & 5 & 20 \\
\hline Gamma Knife & 6 & 4 & 10 \\
\hline Percutaneous & 8 & 2 & 10 \\
\hline Total & 29 & 11 & 40 \\
\hline \multicolumn{4}{|l|}{ Distribution of pain (no., expected $\chi^{2}$ value) } \\
\hline V1 & $7,6.6$ & $1,1.4$ & 8 \\
\hline V1, V2 & $15,15.8$ & $4,3.2$ & 19 \\
\hline V2 & $39,36.6$ & $5,7.4$ & 44 \\
\hline V2, V3 & $33,33.2$ & $7,6.8$ & 40 \\
\hline V3 & $29,30.8$ & $8,6.2$ & 37 \\
\hline V1-V3 & 5,5 & 1,1 & 6 \\
\hline Total & 129 & 27 & 156 \\
\hline$p$ value ( $\chi^{2}$ value $)$ & & & $0.86(1.90)$ \\
\hline \multicolumn{4}{|l|}{ Response to AEDs* (no., expected $\chi^{2}$ value) } \\
\hline No response & $21,22.3$ & $6,4.7$ & 27 \\
\hline Response & $108,106.7$ & $21,22.3$ & 129 \\
\hline Total & 129 & 27 & 156 \\
\hline$p$ value ( $\chi^{2}$ value $)$ & & & $0.46(0.55)$ \\
\hline
\end{tabular}

* Carbamazepine or gabapentin.

drugs (AEDs) was similar between groups. Patients without NVC trended toward a shorter average symptom duration before presentation $(\mathrm{p}=0.07)$; there was a trend toward female predominance in patients without NVC $(\mathrm{p}=$ $0.08)$. Patients without NVC (29.6\%) had undergone more procedures prior to presentation to OHSU than patients with NVC $(14 \%)(\mathrm{p}=0.05)$.

\section{Effect of NVC on Age at Onset}

Average age at onset for patients in whom MRI/MR angiography showed NVC $(\mathrm{n}=158)$ was 51.7 years; the average age at onset in those without NVC $(n=61)$ was 46.5 years, which was significant (Student t-test, $\mathrm{p}=0.02$ ).
A similarly significant difference was noted for median age at onset (Wilcoxon's rank-sum test, $\mathrm{p}=0.03$ ).

Of the 156 patients with TN-I who underwent posterior fossa exploration and MVD or IN, age at onset of TN symptoms in patients without NVC was significantly younger than that of patients with NVC (Table 2). Mean age at onset was 51 and 42.6 years in patients with and without NVC (Student t-test, $\mathrm{p}=0.01$ ), respectively. The difference in mean age at onset was 8.48 years. Power calculations show that the sample size was sufficient to yield a difference of 8.2 years for this measure.

The distribution of age at onset in TN patients with NVC or without NVC was not normal, with the onset of symptoms most frequently occurring in the late 50 s for 
TABLE 2. Effect of NVC on age at symptom onset and symptom duration before surgery in TN

\begin{tabular}{|c|c|c|c|}
\hline Age \& Symptom Duration & w/ NVC & w/o NVC & Total \\
\hline \multicolumn{4}{|l|}{ Age at presentation (yrs) } \\
\hline Mean & 57.1 & 46.2 & $p=0.00$ \\
\hline Median & 58.0 & 47.0 & $p=0.00$ \\
\hline Range & $24.0-82.0$ & $23.0-81.0$ & \\
\hline Total & Mean $55.2 \pm 13.9$ & Median 56.0 & Range 23-82 \\
\hline \multicolumn{4}{|l|}{ Age at onset (yrs) } \\
\hline Mean & 51 & 42.6 & $p=0.01$ \\
\hline Median & 53.3 & 41.2 & $p=0.00$ \\
\hline Range & $9-79$ & $18-79.8$ & \\
\hline Total & Mean $49.6 \pm 14.9$ & Median 51.6 & Range 9-79.8 \\
\hline \multicolumn{4}{|l|}{ Onset age ( $n$, expected $\chi^{2}$ value) } \\
\hline$\leq 35 \mathrm{yrs}$ & $24,28.1$ & $10,5.9$ & 34 \\
\hline$>35$ yrs & $105,100.9$ & $17,21.1$ & 122 \\
\hline Total & 129 & 27 & 156 \\
\hline Patients w/ onset age $\leq 35$ yrs ( $\%)$ & 18.6 & 37 & 21.8 \\
\hline$p$ value ( $\chi^{2}$ value $)$ & & & $0.03(4.45)$ \\
\hline \multicolumn{4}{|l|}{ Symptom duration (mos) } \\
\hline Mean & 77.0 & 43.3 & $p=0.04$ \\
\hline Median & 48 & 36 & $p=0.11$ \\
\hline Total & Mean $70.1 \pm 77.7$ & Median 48 & Range 2-450 \\
\hline
\end{tabular}

patients of both populations. However, a normalized histogram of age at onset in the populations with and without NVC revealed a bimodal distribution of patient age in patients without NVC (Fig. 1). A greater percentage of patients without NVC have symptom onset prior to the age of 35 years, nearly twice that of patients with NVC (with vs without NVC: $18.6 \%$ vs $37 \%$; chi-square, $p=0.03$ ). Importantly, the empirical cumulative distribution of age at onset shows that patients without NVC present significantly earlier than those with NVC (Fig. 2). The distributions are significantly different (2-sample Kolmogorov-Smirnov test, $\mathrm{p}=0.003)$. These are analogous to Kaplan-Meier survival curves (Fig. 3), which show significantly different hazard ratios for patients with versus without NVC ( 0.19 vs 0.35 ; log-rank test, $\mathrm{p}=0.03)$. A similar trend for younger presentation in patients without NVC is noted using median age (with vs without NVC: 53.3 vs 41.2 years; Wilcoxon's rank-sum test, $\mathrm{p}=0.00$ ).

Multivariate analysis was performed using a Cox proportional hazards model. Factors examined included presence of NVC, sex, side of presentation, branch of nerve affected, and response to AEDs. Presence of NVC was significantly associated with later age at onset of $\mathrm{TN}$ symptoms (HR 0.56; $\mathrm{p}=0.01)$. No other factors were significant (Table 3 ).

\section{Effect of NVC on Duration of Symptoms Prior to First Intervention}

To more accurately evaluate the effect of NVC on duration of symptoms prior to intervention, patients with no prior treatments were considered. This eliminates patients who have had symptoms recur prior to presentation at OHSU. There were a total of 122 patients with TN-I presenting for a first surgical intervention. Of these, 103 $(84 \%)$ had NVC and $19(16 \%)$ did not.

On average, patients without NVC undergo surgical treatment (MVD or IN) after a shorter duration of symptoms compared with patients with NVC, 43.3 months versus 77 months, respectively (Fig. 4). Kaplan-Meier analysis confirms that this tendency for earlier treatment is significant (log-rank test, $\mathrm{p}=0.02$ ).

Multivariate analysis was performed to examine the effect of the following factors on symptom duration: age at onset, presence of NVC, sex, side of presentation, response to AEDs, and distribution of symptoms (Table 3). Interestingly, age at onset interacted significantly but only slightly with duration of symptoms (HR 10.3, p = 0.00). As expected, absence of NVC was associated with shorter duration of symptoms prior to intervention $(\mathrm{HR} 0.56, \mathrm{p}=$ $0.01)$. Analysis of variance was performed to further characterize the effect of age and NVC on symptom duration. There was an effect of age at onset when the patient was less than 35 years $(\mathrm{F}=4.53, \mathrm{p}=0.04)$ and in the presence of NVC $(\mathrm{F}=7.81, \mathrm{p}=0.01)$, and a significant interaction existed between these factors $(F=4.84, p=0.03)$. Post hoc testing showed that younger patients with NVC have a longer duration of symptoms (Student t-test, all $\mathrm{p}<0.01$, Bonferroni-corrected), while all other cell means were not significantly different (Fig. 5).

\section{NVC and Reoperation}

Patients with TN without NVC were treated with IN during posterior fossa exploration while patients with NVC were treated with MVD. Chart review identified pa- 


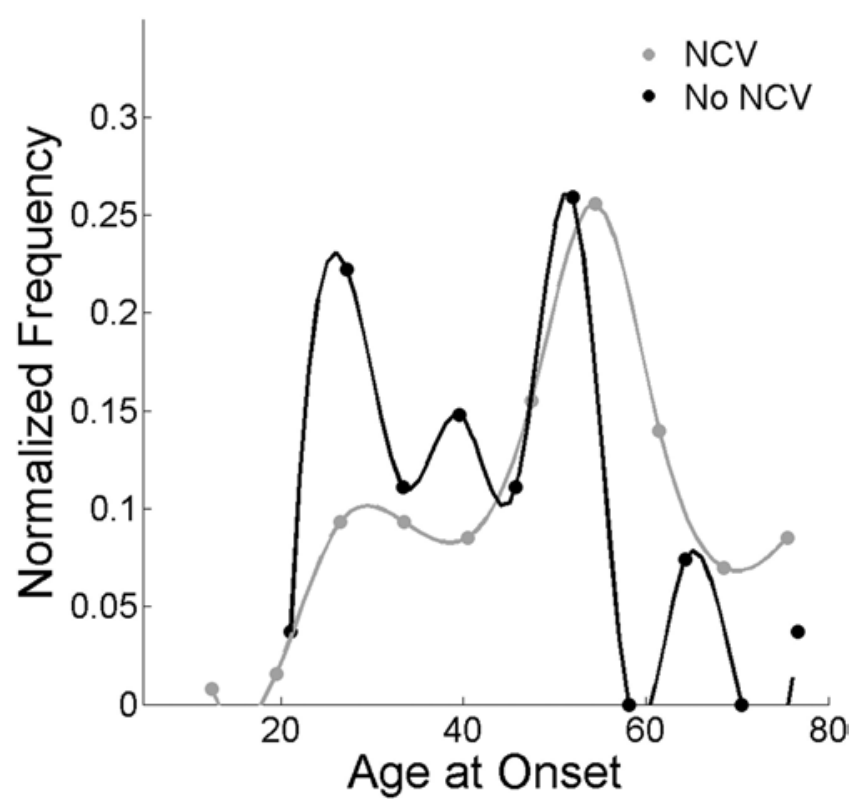

FIG. 1. Histogram showing age at symptom onset of TN in patients with (gray line) and without (black line) NVC. Onset age appears to have a bimodal distribution in patients without NVC, with an average age of presentation that is significantly younger than those with NVC $(p=0.007)$. The histogram is smoothed with a cubic spline to aid visualization.

tients who required reoperation for recurrence of symptoms after these treatments; patients with previous procedures were excluded as described above. Reexploration or treatment with radiofrequency ablation was performed. These cases were classified as treatment failure.

Median follow-up time was 48.8 months for all patients. Kaplan-Meier analysis showed no significant difference between patients with and without NVC (Fig. 6). There was no difference in patient response to IN (without NVC) versus MVD (with NVC) as measured by reoperation rate (log-rank test, $\mathrm{p}=0.55$ ).

\section{Discussion}

The natural symptomatic history of TN is well defined, with trigeminal nerve compression described as the most common factor associated with its etiology..$^{1,4,13}$ However, since the 1930s, it has been recognized that there are TN patients who present with a well-defined symptomatic history but no NVC. ${ }^{6,19}$ MVD, when possible, is unequivocally the most effective and durable treatment for TN. . $^{1,432}$ However, MVD is not possible if there is no evidence of NVC. Fortunately, TN without NVC can be treated effectively, and deafferentation procedures, from percutaneous surgery to radiosurgery, are effective and safe. When posterior fossa exploration for TN reveals no NVC, treatment involves selectively destructive procedures such as partial sensory rhizotomy, trigeminal root compression, or IN procedure. Unfortunately, the long-term pain relief of these treatments is not as satisfactory as that of MVD. ${ }^{5,14,33-36}$ Immediate outcomes from these procedures are similar to those of MVD; however, over the long term, recurrence rates appear to be higher for patients without NVC, regardless of treatment. $22,28,29,35$ Additionally, recur-

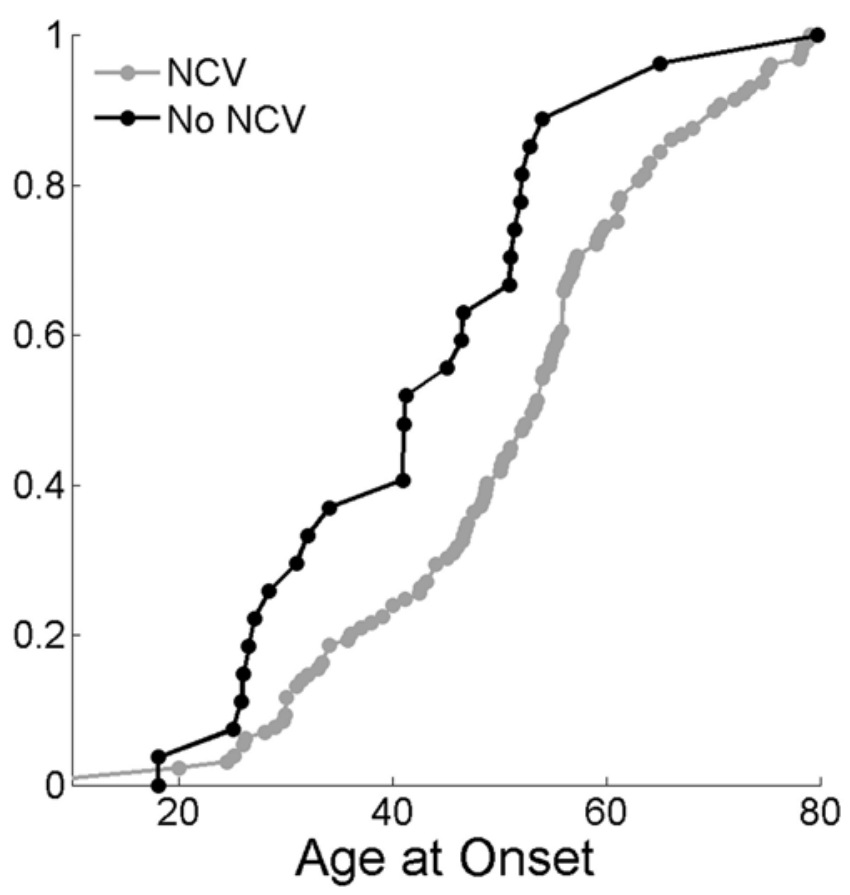

FIG. 2. The empirical cumulative distribution of age at TN symptom onset in patients with (gray line) and without (black line) NVC shows that a greater proportion of patients without NVC present earlier than those with NVC $(p=0.002)$.

rence of $\mathrm{TN}$ takes place without recurrence of $\mathrm{NVC},{ }^{19}$ and cases refractory to MVD are notoriously difficult to treat. $^{27}$

In the current study we examined characteristics of TN-I patients with and without NVC. We demonstrate that the age distribution of these 2 populations was significant-

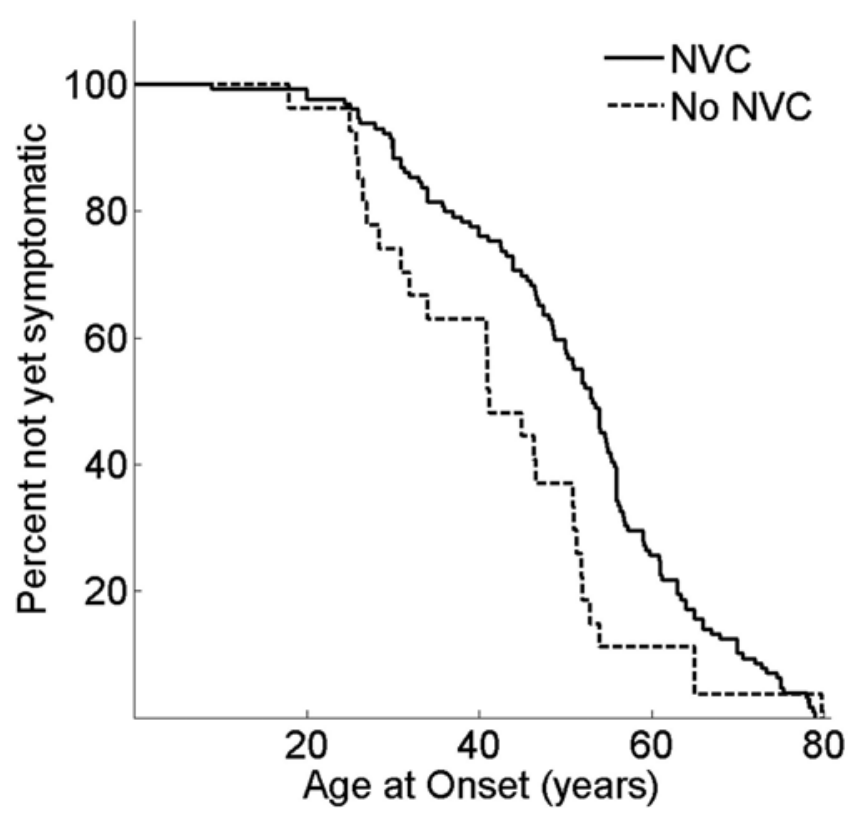

FIG. 3. Kaplan-Meier curves for onset at TN symptom onset in patients with NVC (solid line) and without NVC (dashed line). Log-rank test shows a significant difference in hazard ratio between the 2 groups (NVC vs no NVC: 0.19 vs 0.35 ; log-rank test, $p=0.03$ ). 
TABLE 3. Factors affecting age of onset and duration of symptoms prior to treatment

\begin{tabular}{lrrrr}
\hline \multicolumn{1}{c}{ Factor } & Coefficient & $\begin{array}{c}\text { Standard } \\
\text { Error }\end{array}$ & HR & p Value \\
\hline Age at onset & & & & \\
\hline NVC & -0.5811 & 0.2311 & 0.5593 & 0.01 \\
\hline Female sex & 0.0937 & 0.1755 & 1.0982 & 0.59 \\
\hline Response to AEDs & 0.1152 & 0.2204 & 1.1221 & 0.60 \\
\hline Rt-side symptoms & 0.2819 & 0.1789 & 1.3257 & 0.11 \\
\hline V1 & -0.4023 & 0.2476 & 0.6688 & 0.10 \\
\hline V2 & 0.3234 & 0.2120 & 1.3818 & 0.12 \\
\hline V3 & -0.3381 & 0.2150 & 0.7131 & 0.11 \\
\hline Duration of symptoms & & & & \\
\hline Age at onset & 0.0288 & 0.0065 & 1.0292 & 0.00 \\
\hline NVC & -0.5827 & 0.2331 & 0.5584 & 0.01 \\
\hline Female sex & 0.3038 & 0.1759 & 1.3550 & 0.08 \\
\hline Response to AEDs & 0.2271 & 0.2284 & 1.2549 & 0.32 \\
\hline Rt-side symptoms & -0.1838 & 0.1914 & 0.8321 & 0.33 \\
\hline V1 & 0.1849 & 0.2189 & 1.2030 & 0.39 \\
\hline V2 & -0.3110 & 0.2057 & 0.7327 & 0.13 \\
\hline V3 & -0.1163 & 0.1887 & 0.8902 & 0.53 \\
\hline Cant & & & &
\end{tabular}

* Carbamazepine or gabapentin.

ly different; Kaplan-Meier analysis revealed that onset of symptoms in patients without NVC occurred at a younger age. A frequency histogram revealed that for patients without NVC, age at onset of symptoms was bimodal, with a sizeable subset of patients presenting before 35 years of age.

The failure rate of treatment with IN did not differ significantly from that of treatment with MVD. However, results were obtained from chart review only and did not measure recurrence of symptoms. Long-term results for IN have recently been studied, and findings suggest that success with this treatment strategy for patients without NVC may be more durable than other ablative procedures. $^{18}$ This may be particularly relevant for younger patients, as ablative procedures are less durable than MVD and IN. For example, median time to pain recurrence for percutaneous radiofrequency trigeminal gangliolysis is on the order of 3 years, ${ }^{5}$ while median time to treatment failure was not reached after 5 years for IN. ${ }^{18}$ A structured, well-designed comparison of the efficacy and durability of different techniques for treating TN without NVC is warranted, and acquiring a sufficient cohort for a prospective trial would require a concerted, collaborative effort. The findings of this study provide a rationale for pursuing such an effort: if patients without NVC tend to be younger and likelihood of recurrence is higher, then these patients have a greater chance of being subject to repeated ablative procedures, with concomitantly increased chances of the sequelae of deafferentation.

The findings of this study also reinforce the notion that some factor other than NVC may prompt development or recurrence of $\mathrm{TN}$, and raise the question of whether patients with TN without NVC represent a distinct population, with differing clinical or pathological characteristics

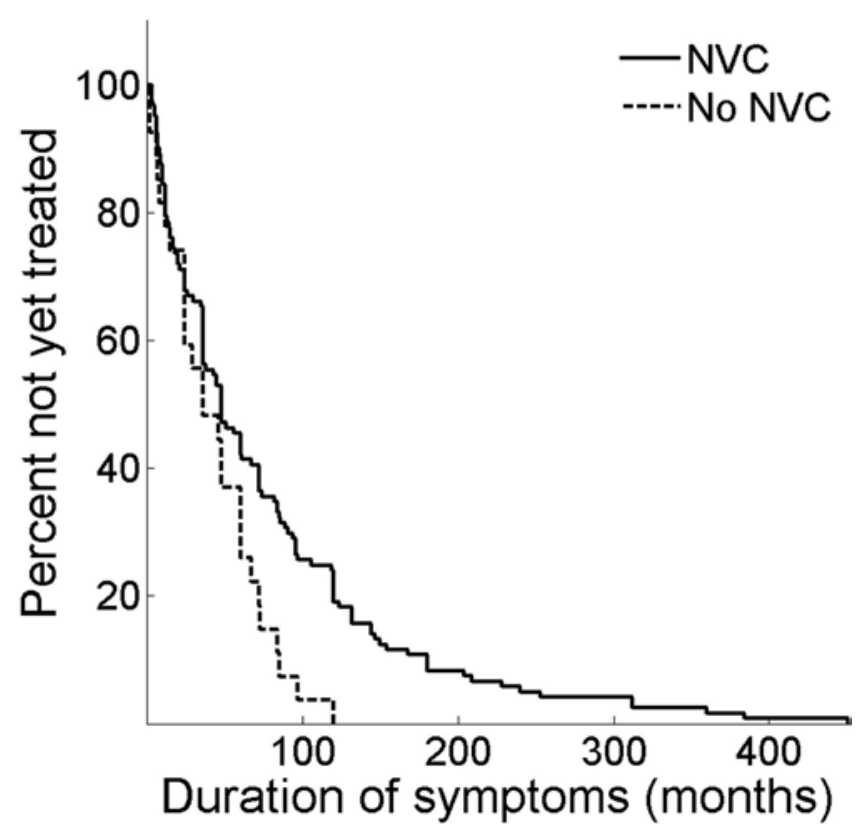

FIG. 4. Kaplan-Meier curves in patients who underwent a first surgical treatment for TN show that patients without NVC (dashed line) were treated sooner than those with NVC (solid line). Symptom duration differed between patients with NVC and without NVC (log-rank test: $p=$ 0.02 ).

from those with NVC. A trend toward female predominance existed in the population of patients without NVC. In our study, the proportion of female to male patients without NVC was 2.9:1; however, other studies have reported more dramatic findings, with 6:1 and even 20:1 ratios. ${ }^{28,29}$ Also, the presence or absence of NVC was found to affect symptom duration before initial surgical treatment.

The effect of NVC on symptom duration is difficult to interpret as it seems to differentially affect patients based on age. This too may have implications regarding the pathogenesis and natural history of TN. Younger patients with NVC seem to endure symptoms longer than older patients or patients without NVC. This could be attributed to any of several factors. It does not seem to be related to initial response to medical treatment as we found no significant difference in initial response to AEDs between young patients with NVC versus older patients with NVC or patients without NVC (chi-square $=1.60, p=0.66$ ). It is possible that AED side effects are better tolerated in younger patients, and symptom duration may reflect a longer duration of medical treatment. The longer duration of symptoms in younger patients may also reflect delays in diagnosis as TN is less common in this age group; however, one would expect that this would affect patients with and without NVC equally. An alternative and more likely explanation is that the finding may reflect the natural history of $\mathrm{TN}$, with remissions being common initially but increasing in frequency and length as the disease progresses; this progression may be more precipitous in patients without NVC. More specifically, younger patients with TN with NVC may have a more indolent course in comparison with younger patients without NVC, who thus present for surgical treatment sooner. 


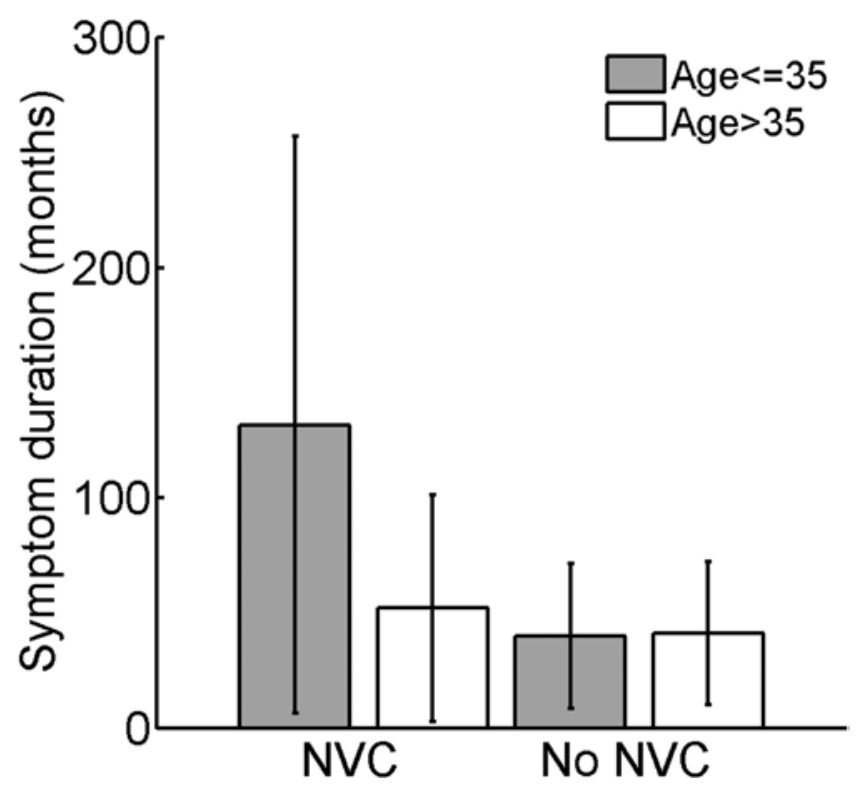

FIG. 5. Age at onset and NVC affect the duration of symptoms prior to first intervention for TN. Of patients with NVC, those younger than 35 years of age (gray bar) tended to have a longer duration of symptoms than those older than 35 years of age (white bar). This effect is not seen in patients without NVC. ANOVA shows main effects of NVC $(p=0.01)$ and age at onset $(p=0.04)$ on symptom duration but also a significant interaction between the 2 factors $(p=0.03)$; post hoc analysis of cell means show that young patients with NVC have significantly longer duration of symptoms than other groups $(p<0.001$, Bonferroni-corrected for 6 comparisons).

$\mathrm{TN}$ is known to be a heterogeneous disease; previous studies have reported that TN-II, for example, presents in younger patients with a female predilection, findings that correlate with response to MVD. ${ }^{24}$ The presence or absence of NVC has been shown to be a prognostic factor for response to MVD. ${ }^{1}$ The findings in this study support the notion that TN-I with and without NVC exhibit a distinctly different set of clinical and pathological entities. Clinical evidence is represented by a significant difference in age at onset of TN in patients without NVC; the latter is implicit in the lack of vascular compromise of the REZ of the trigeminal nerve.

However, this does not exclude the possibility of a shared pathophysiological model underlying TN with or without NVC. Another interpretation of these results is that $\mathrm{NVC}$ is not the primary cause of TN, but rather an associated feature in older patients; NVC may not be required for development of TN at any age, but it may be more likely with advancing age. Devor's "ignition" hypothesis for the pathogenesis of TN, for example, posits a dual pathology underlying the development of $\mathrm{TN}$, in which injury to nerve axons leads to synchronized, abnormal activity within the cell bodies in the trigeminal ganglion, evoking the painful paroxysms of the disease. ${ }^{8}$ Histopathology of nerve root specimens from patients with NVC show demyelinating or dysmyelinating pathology near the compressing vessel, whereas specimens from patients without NVC show only normal age-related changes. ${ }^{8,9}$ Similar changes in myelination are seen in electron micrographs of the trigeminal ganglion in patients with TN. ${ }^{2,15,16}$ Alternately, a

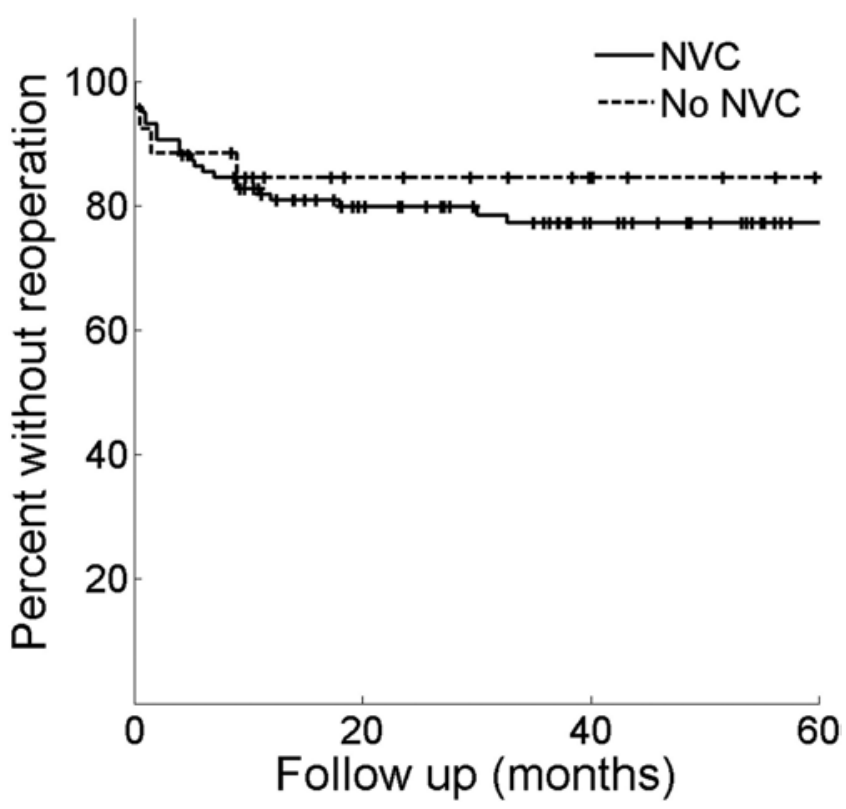

FIG. 6. Failure rate measured as the need for reoperation did not differ for TN patients with or without NVC. Kaplan-Meier analysis shows that treatment with IN in cases without NVC (dashed line) did not fail at a higher rate than treatment with MVD in cases with NVC (solid line) (logrank test: $p=0.55)$.

case for a central etiology of TN was put forth by King in 1967, who presented evidence of abnormalities in the efferent inhibitory mechanisms within the brainstem at the nucleus caudalis. ${ }^{17}$ Both of these theories could account for development of TN without NVC, whether it is from aberrant activity within the trigeminal ganglion or abnormal efferent reflex activity within the brainstem.

More recent examinations of the microstructure of the trigeminal system in TN using diffusion-tensor imaging likewise show pathology at the level of the peripheral and central nervous system. These changes were present in the affected side and not the unaffected side, and are not seen in normal control subjects. ${ }^{7,20}$ These measures are thought to reflect microstructural evidence of demyelination, edema, or neuroinflammation.

Changes in white matter diffusivity were also observed in the corpus callosum, cingulum, corona radiata, and superior longitudinal fasciculus. ${ }^{7}$ These findings suggest widespread changes in white matter tracts involved in sensory and cognitive-affective aspects of pain, and have been seen in patients suffering from chronic pain due to irritable bowel syndrome ${ }^{10}$ spinal cord injury ${ }^{11}$ phantom limb, ${ }^{31}$ fibromyalgia, ${ }^{21}$ and poststroke pain. ${ }^{30}$

In other words, patients with TN may have a predilection for development of this pain syndrome, which is distinct from the presence of NVC but may reflect some susceptibility to alterations in myelin at the level of the peripheral nerve, in the trigeminal ganglion or REZ. Structural factors in the central nervous system may also contribute to this pain, whether at the brainstem or in more widely distributed networks such as those involved in processing sensorimotor data, coordinating attention and memory, or supporting the cognitive-affective aspects of pain. 
It is important to note that patients diagnosed with TN-I do not have MS, ipso facto. Either a history of MS or waxing and waning neurological symptoms suspicious for MS would prompt a diagnosis of symptomatic TN or referral for definitive diagnostic testing, respectively. Importantly, no patients with symptomatic TN are treated via craniotomy at our institution. None of the patients without NVC presented with other symptoms of MS, nor did they have MRI findings compatible with widespread demyelinating disease. Symptomatic TN was never found to be the initial presenting symptom of a patient with MS during the study period.

There are study limitations. It is a retrospective review and therefore subject to bias. In particular, because all patients in this study underwent preoperative imaging that has a high sensitivity and specificity for detecting NVC, there exists the confounder that older patients without detectable NVC may be more likely to select percutaneous treatment over posterior fossa exploration. The decision to include only those patients whose status vis-à-vis NVC was confirmed at operation, and therefore more accurately confirmed than by imaging alone, might in turn artificially reflect a younger population.

There are 2 facts that mitigate this bias: First, only patients considered candidates for MVD were imaged preoperatively. Older patients and patients with severe medical comorbidities were not considered for MVD; this criteria was applied prior to obtaining high-resolution images. In other words, MRI findings were not used to guide treatment recommendations vis-à-vis craniotomy versus percutaneous treatment. In fact, at our institution, craniotomy to perform IN is routinely performed in patients without NVC. Bias imposed by the predilection against craniotomy in elderly patients would be present regardless of imaging findings, and thus the impact of selection bias is likely less than expected.

Second, as determined by imaging alone, examination of all TN-I patients $(n=219)$, regardless of eventual treatment, revealed a similar trend toward younger age in patients without NVC $(n=61)$ compared with those with NVC $(n=158)$. The statistically significant correlation between the absence of NVC and younger age at onset is preserved when eventual treatment is discounted. Notably, the average age at onset of both groups was higher than that of patients undergoing posterior fossa exploration. This should not be surprising, given that a similar aversion to craniotomy should exist to a certain extent for older patients with or without NVC. Again, because MRI was performed only in patients considered candidates for MVD, selection bias should be mitigated.

While the sample size in this study is sufficient to show statistically significant results, the number of patients without NVC is still relatively small $(n=27)$. Including patients without intraoperative confirmation of NVC would increase this number significantly at the cost of introducing additional confounding factors. Therefore, we limited more detailed analysis to confirmed cases of NVC.

Examination of the duration of symptoms in this study is also somewhat cursory in nature. There is no measure of baseline severity of symptoms, and, as mentioned above, the results are difficult to interpret in light of interactions between multiple factors. Several sources of bias leading to longer duration of symptoms prior to treatment are not controlled. This factor also relies solely on patient reporting, which can be unreliable. Examining only patients undergoing posterior fossa surgery also introduces bias. Such shortcomings are, however, shared with any large observational study reporting statistics regarding duration of symptoms. . $^{1,32}$

As noted before, the examination of outcomes in this study is not comprehensive. Reoperation is not a very reliable indicator of recurrence of TN but is of interest in and of itself; determining failure rates by chart review alone is also an inadequate measure of outcomes. However, a thorough analysis of recurrence rates was not the intent of this paper.

\section{Conclusions}

TN presents with or without NVC. Patients with TN-I and without NVC are younger at onset of symptoms than patients with NVC. Factors such as sex, laterality and distribution of symptoms, and response to AEDs are not related to age at onset. A longer duration of symptoms appears to be related to the presence of NVC in patients with onset of symptoms before the age of 35 years. These findings suggest that TN patients without NVC form a distinct subset of patients. Further work to characterize the clinical and pathophysiological implications of this distinction should follow.

\section{References}

1. Barker FG II, Jannetta PJ, Bissonette DJ, Larkins MV, Jho HD: The long-term outcome of microvascular decompression for trigeminal neuralgia. N Engl J Med 334:1077-1083, 1996

2. Beaver DL: Electron microscopy of the Gasserian ganglion in trigeminal neuralgia. J Neurosurg 26:138-150, 1967

3. Burchiel KJ: A new classification for facial pain. Neurosurgery 53:1164-1167, 2003

4. Burchiel KJ, Clarke H, Haglund M, Loeser JD: Long-term efficacy of microvascular decompression in trigeminal neuralgia. J Neurosurg 69:35-38, 1988

5. Burchiel KJ, Steege TD, Howe JF, Loeser JD: Comparison of percutaneous radiofrequency gangliolysis and microvascular decompression for the surgical management of tic douloureux. Neurosurgery 9:111-119, 1981

6. Dandy WE: Concerning the cause of trigeminal neuralgia. Am J Surg 24:447-455, 1934

7. DeSouza DD, Hodaie M, Davis KD: Abnormal trigeminal nerve microstructure and brain white matter in idiopathic trigeminal neuralgia. Pain 155:37-44, 2014

8. Devor M, Amir R, Rappaport ZH: Pathophysiology of trigeminal neuralgia: the ignition hypothesis. Clin J Pain 18:4-13, 2002

9. Devor M, Govrin-Lippmann R, Rappaport ZH: Mechanism of trigeminal neuralgia: an ultrastructural analysis of trigeminal root specimens obtained during microvascular decompression surgery. J Neurosurg 96:532-543, 2002

10. Ellingson BM, Mayer E, Harris RJ, Ashe-McNally C, Naliboff BD, Labus JS, et al: Diffusion tensor imaging detects microstructural reorganization in the brain associated with chronic irritable bowel syndrome. Pain 154:1528-1541, 2013

11. Gustin SM, Wrigley PJ, Siddall PJ, Henderson LA: Brain anatomy changes associated with persistent neuropathic pain following spinal cord injury. Cereb Cortex 20:1409-1419, 2010 
12. Headache Classification Committee of the International Headache Society (IHS): The International Classification of Headache Disorders, 3rd edition (beta version). Cephalalgia 33:629-808, 2013

13. Jannetta PJ: Arterial compression of the trigeminal nerve at the pons in patients with trigeminal neuralgia. J Neurosurg 26:159-162, 1967

14. Kanpolat Y, Savas A, Bekar A, Berk C: Percutaneous controlled radiofrequency trigeminal rhizotomy for the treatment of idiopathic trigeminal neuralgia: 25-year experience with 1,600 patients. Neurosurgery 48:524-534, 2001

15. Kerr FWL: Correlated light and electron microscopic observations on the normal trigeminal ganglion and sensory root in man. J Neurosurg 26:132-137, 1967

16. Kerr FWL: Pathology of trigeminal neuralgia: light and electron microscopic observations. J Neurosurg 26:151-156, 1967

17. King RB: Evidence for a central etiology of tic douloureux. $\mathbf{J}$ Neurosurg 26:175-180, 1967

18. Ko AL, Ozpinar A, Lee A, Raslan A, McCartney S, Burchiel KJ: Long-term efficacy and safety of internal neurolysis for trigeminal neuralgia without vascular compression. J Neurosurg [epub ahead of print February 13, 2015 DOI: 10.3171/2014.12.JNS14469]

19. Lee A, McCartney S, Burbidge C, Raslan AM, Burchiel KJ: Trigeminal neuralgia occurs and recurs in the absence of neurovascular compression. Clinical article. J Neurosurg 120:1048-1054, 2014

20. Liu Y, Li J, Butzkueven H, Duan Y, Zhang M, Shu N, et al: Microstructural abnormalities in the trigeminal nerves of patients with trigeminal neuralgia revealed by multiple diffusion metrics. Eur J Radiol 82:783-786, 2013

21. Lutz J, Jäger L, de Quervain D, Krauseneck T, Padberg F, Wichnalek M, et al: White and gray matter abnormalities in the brain of patients with fibromyalgia: a diffusion-tensor and volumetric imaging study. Arthritis Rheum 58:3960-3969, 2008

22. Ma Z, Li M: "Nerve combing" for trigeminal neuralgia without vascular compression: report of 10 cases. Clin J Pain 25:44-47, 2009

23. McCartney S, Weltin M, Burchiel KJ: Use of an artificial neural network for diagnosis of facial pain syndromes: an update. Stereotact Funct Neurosurg 92:44-52, 2014

24. Miller JP, Acar F, Burchiel KJ: Classification of trigeminal neuralgia: clinical, therapeutic, and prognostic implications in a series of 144 patients undergoing microvascular decompression. J Neurosurg 111:1231-1234, 2009

25. Miller JP, Acar F, Hamilton BE, Burchiel KJ: Radiographic evaluation of trigeminal neurovascular compression in patients with and without trigeminal neuralgia. J Neurosurg 110:627-632, 2009

26. Miller JP, Magill ST, Acar F, Burchiel KJ: Predictors of longterm success after microvascular decompression for trigeminal neuralgia. J Neurosurg 110:620-626, 2009

27. Pollock BE, Stein KJ: Surgical management of trigeminal neuralgia patients with recurrent or persistent pain despite three or more prior operations. World Neurosurg 73:523528,2010
28. Revuelta-Gutiérrez R, López-González MA, Soto-Hernández JL: Surgical treatment of trigeminal neuralgia without vascular compression: 20 years of experience. Surg Neurol 66:32-36, 2006

29. Revuelta-Gutierrez R, Martinez-Anda JJ, Coll JB, CamposRomo A, Perez-Peña N: Efficacy and safety of root compression of trigeminal nerve for trigeminal neuralgia without evidence of vascular compression. World Neurosurg 80:385-389, 2013

30. Seghier ML, Lazeyras F, Vuilleumier P, Schnider A, Carota A: Functional magnetic resonance imaging and diffusion tensor imaging in a case of central poststroke pain. J Pain 6:208-212, 2005

31. Simões EL, Bramati I, Rodrigues E, Franzoi A, Moll J, Lent $\mathrm{R}$, et al: Functional expansion of sensorimotor representation and structural reorganization of callosal connections in lower limb amputees. J Neurosci 32:3211-3220, 2012

32. Sindou M, Leston J, Decullier E, Chapuis F: Microvascular decompression for primary trigeminal neuralgia: long-term effectiveness and prognostic factors in a series of 362 consecutive patients with clear-cut neurovascular conflicts who underwent pure decompression. J Neurosurg 107:1144-1153, 2007

33. Verheul JB, Hanssens PE, Lie ST, Leenstra S, Piersma H, Beute GN: Gamma Knife surgery for trigeminal neuralgia: a review of 450 consecutive cases. J Neurosurg 113 Suppl:160-167, 2010

34. Young B, Shivazad A, Kryscio RJ, St Clair W, Bush HM: Long-term outcome of high-dose Gamma Knife surgery in treatment of trigeminal neuralgia. Clinical article. J Neurosurg 119:1166-1175, 2013

35. Young JN, Wilkins RH: Partial sensory trigeminal rhizotomy at the pons for trigeminal neuralgia. J Neurosurg 79:680687,1993

36. Zakrzewska JM, Lopez BC, Kim SE, Coakham HB: Patient reports of satisfaction after microvascular decompression and partial sensory rhizotomy for trigeminal neuralgia. Neurosurgery 56:1304-1312, 2005

\section{Author Contributions}

Conception and design: Burchiel. Acquisition of data: Burchiel, Ko, Lee, Ozpinar. Analysis and interpretation of data: Burchiel, Ko, Lee, Raslan, Ozpinar. Drafting the article: Ko, Lee. Critically revising the article: Burchiel, Ko, Raslan, McCartney. Reviewed submitted version of manuscript: all authors. Approved the final version of the manuscript on behalf of all authors: Burchiel. Statistical analysis: Ko. Administrative/technical/material support: McCartney. Study supervision: Burchiel. IRB preparation and submission: McCartney.

\section{Correspondence}

Kim J. Burchiel, Department of Neurological Surgery, Oregon Health \& Science University, mail code: CH8N, 3303 SW Bond Ave., Portland, OR 97239. email: burchiek@ohsu.edu. 